\title{
Flore associée aux péricoronarites des troisièmes molaires mandibulaires. Composition et sensibilité aux antibiotiques
}

\author{
Microflora of mandibular third molars pericoronitis. \\ Composition and susceptibility to antibiotics
}

\author{
JEAN-LOUIS SIXOU*, CHRISTOPHE MAGAUD**, ANNE JOLIVET-GOUGEON***, \\ MARTINE BONNAURE-MALLET ${ }^{\star \star \star}$, MICHEL CORMIER ${ }^{\star \star \star}$
}

\begin{abstract}
RÉSUMÉ
Cette étude a eu pour objectif d'évaluer par culture la composition de la flore associée aux péricoronarites des troisièmes molaires mandibulaires et d'étudier sa sensibilité aux antibiotiques le plus souvent prescrits en France. Résultats : la flore isolée était hétérogène, le plus souvent polymicrobienne et mixte aérobie/anaérobie. La flore anaérobie était prédominante dans la plupart des cas. Les anaérobies stricts ont été retrouvés dans 53 des 61 échantillons. Les bactéries le plus souvent isolées ont été les streptocoques viridans (58/61), puis des genres anaérobies (Prevotella, Veillonella) ou à croissance préférentielle en anaérobie (Actinomyces, Capnocytophaga). L'amoxicilline est l'antibiotique le plus actif sur la flore totale et notamment les germes aérobies, mais peut favoriser la sélection de microorganismes résistants, en particulier ceux producteurs de bêtalactamases. Le métronidazole seul ou en association à la spiramycine a été le plus efficace sur les bactéries anaérobies strictes et le moins agressif pour la flore normale de la cavité buccale. Son utilisation est intéressante dans les cas de péricoronarites avec une flore mixte aérobie/anaérobie bien documentée. (Med Buccale Chir Buccale 2004; 10: 11-20)
\end{abstract}

mots clés : péricoronarite, microbiologie, antibiotique, amoxicilline, spiramycine, métronidazole

\section{SUMMARY}

The aim of this investigation was to evaluate by culture the predominant flora associated with mandibular third molar pericoronitis and to investigate its susceptibility to antibiotics most regularly prescribed by French clinicians. Results: The flora found was heterogeneous, usually polymicrobial and mixed aerobic/anaerobic. In most of cases the flora cultivated in anaerobic condition was predominant. Obligate anaerobes were found in 53 out of 61 samples. The bacteria most commonly detected were viridans streptococci (58/61) [including S. anginosus (34) and S. oralis (30) groups], strict anaerobes and bacteria growing preferentially under anaerobic condition [Genera Prevotella (36), Actinomyces (36), Canocytophaga (29), Veillonella (26)]. Amoxicillin and pristinamycin were found to be the most effective against the flora tested, particularly aerobic organisms. Beta-lactamase producing strains were

* Equipe de Biologie Buccale UPRES-EA 1256 et Département d'Odontologie Pédiatrique, U.F.R. d'Odontologie, Université de Rennes 1

** Equipe de Biologie Buccale UPRES-EA 1256, U.F.R. d'Odontologie, Université de Rennes 1

*** Laboratoire de Microbiologie UPRES-EA 1254, U.F.R. de Pharmacie, Université de Rennes 1

Crédits de recherche:

Fondation Langlois, Conseil Général d'Ille-et-Vilaine, Laboratoire Aventis. Remerciements à Hélène Pinsard-Solhi, Xavier Moisan, Carine Desoindre, Noël Grosset, Céline Allaire pour leur assistance technique et éditoriale.

Demande de tirés à part :

Dr Jean-Louis SIXOU 2 place Pasteur 35000 Rennes France 
médecine

buccale

chirurgie

buccale

VOL. $10, \mathrm{~N}^{\circ} 1$ 2004

page 12

detected in respectively $34.6 \%$ and $14 \%$ of the samples using either selective or non-selective media. Metronidazole alone or in combination with spiramycin was the most effective against obligate anaerobic bacteria.

Conclusion: These results highlight the diversity of the microflora associated with pericoronitis and the importance of the anaerobic flora. Amoxicillin is the most powerful antibiotic on this flora but allows the selection of resistant micro-organisms particularly beta-lactamase producing strains. Metronidazole alone or in combination with spiramycin proved the most effective against obligate anaerobic bacteria allowing its use in pericoronitis with a well documented mixed aerobic/anaerobic flora. (Med Buccale Chir Buccale 2004; 10: 11-20)

key words : pericoronitis, microbiology, antibiotics, amoxicillin, spiramycin, metronidazole

Les troisièmes molaires en éruption présentent sur leur face distale une pseudo-poche parodondale plus ou moins profonde susceptible de créer des conditions locales favorables au développement de microorganismes anaérobies stricts ou à croissance anaérobie préférentielle. Cette pseudo-poche est ouverte sur la cavité buccale. Elle peut être colonisée par les bactéries de la salive, lesquelles sont majoritairement aéro-anaérobies. La connaissance de cette flore lors d'épisodes de péricoronarite, ainsi que de sa sensibilité aux antibiotiques les plus fréquemment prescrits en France, est primordiale pour mettre rapidement en place un traitement adapté.

\section{MATÉRIELS ET MÉTHODES}

\section{Collecte et sélection des échantillons}

Un prélèvement a été effectué chez 119 des 322 patients inclus dans un protocole de comparaison de l'efficacité de deux antibiotiques lors du traitement médicamenteux des péricoronarites [1]. Les prélèvements ont été effectués après isolement de la dent à l'aide de rouleaux de coton stérile et d'une aspiration. La surface émergée de la troisième molaire, ainsi que la face distale de la seconde molaire ont été nettoyées. Une pointe de papier stérile (Mynol Fine ${ }^{T M}$ ) a été insérée dans la pseudo-poche pendant 30 secondes, puis placée dans $1,5 \mathrm{ml}$ d'un milieu de transport préréduit (VMGA III) préparé selon Dahlèn et coll. [2]. Les prélèvements ont été traités dans un délai de
24 heures, de façon identique, par l'Equipe de Biologie Buccale de I'UFR d'Odontologie de Rennes (UPRES EA 1256) ou par le Laboratoire de Microbiologie de I'UFR de Pharmacie de Rennes (UPRES EA 1254).

\section{Premier groupe : étude de la flore à l'aide de milieux contenant des anti- biotiques}

Les dilutions, l'ensemencement, l'incubation et les identifications microbiennes ont été effectués selon des techniques standards déjà décrites [3]. Les ensemencements ont été effectués sur gélose Brucella (BBL ${ }^{\mathrm{TM}}$, AES $140072^{\mathrm{TM}}$ ) contenant $5 \%$ de sang de mouton, enrichie en vitamine $\mathrm{K} 1$ ( $1 \mathrm{mg} / \mathrm{L}$ ) et en hémine $(10 \mathrm{mg} / \mathrm{L})$, et sur les mêmes géloses Brucella contenant différents antibiotiques à des concentrations choisies en fonction des CMI de chacun d'entre eux : Amoxicilline (0.5 mg/L) (A1), Amoxicilline (4 mg/L) (A2), Pristinamycine (1mg/l) (P), Spiramycine (1 mg/L) (S), Métronidazole (4 mg/L) (M), Spiramycine + Métronidazole (1 $\mathrm{mg} / \mathrm{L}+4 \mathrm{mg} / \mathrm{L})(\mathrm{SM})$.

La production de bêta-lactamases, par les souches isolées sur milieux contenant de l'amoxicilline, a été évaluée par un test à la nitrocéfine (BBL, Cefinase ${ }^{\mathrm{TM}}$ 231650). Leur sensibilité à l'amoxicilline a ensuite été mesurée, par méthode de dilution en gélose conformément aux recommandations du National Committee for Clinical and Laboratory Standards (NCCLS) (USA) [4] et du Comité de l'Antibiogramme de la Société Française de Microbiologie (SFM) [5]. 
Deuxième groupe : étude de la flore totale puis évaluation de la sensibilité des souches isolées à différents antibiotiques

L'ensemencement, l'identification des micro-organismes et la recherche de bêta-lactamases, ont été effectués de façon identique au groupe précédent, en utilisant des géloses Brucella au sang ne contenant pas d'antibiotiques. L'évaluation de la sensibilité des souches isolées a été effectuée, selon les recommandations du NCCLS et de la SFM, aux concentrations critiques définies par le Comité de l'Antibiogramme de la SFM (Tableau 1).

\section{RÉSULTATS}

\section{Population / Echantillons}

Sur les 119 échantillons, 61 ont été retenus (arrivée dans les délais, absence de contamination avérée ou possible). Ils ont été répartis dans les deux groupes précédemment décrits. Le premier groupe (26/61) comprenait 10 hommes et 16 femmes, âgés de 18 à 30 ans (âge moyen 22,9 ans \pm 3.2 ans). Le deuxième groupe (35/61)

Tableau 1 : Groupe 2 : Concentrations critiques utilisées pour évaluer la sensibilité à différents antibiotiques Group 2 : Critical concentrations used for evaluation of the susceptibility to various antibiotics

$\begin{array}{lcc}\text { Antibiotique } & \begin{array}{c}\text { Concentration } \\ \text { mg/L }\end{array} & \text { Noté } \\ \text { Amoxicilline } & 0,5 & \mathrm{~A} 1 \\ \text { Amoxicilline } & 4 & \mathrm{~A} 2 \\ \text { Spiramycine } & 1 & \mathrm{~S} 1 \\ \text { Spiramycine } & 4 & \mathrm{~S} 2 \\ \text { Métronidazole } & 4 & \mathrm{M} \\ \text { Spiramycine } & & \\ \quad+ & 1+4 & \mathrm{MS} 1 \\ \text { Métronidazole } & & \\ \text { Spiramycine } & & \mathrm{MS} 2 \\ \quad+ & 4+4 & \mathrm{P} 1 \\ \text { Métronidazole } & 1 & \mathrm{P} 2 \\ \text { Pristinamycine } & 2 & \end{array}$

était composé de 20 hommes et 15 femmes, âgés de 18 à 52 ans (âge moyen 26,8 \pm 8,2 ans).

\section{Flore totale}

Dans le groupe 1, les valeurs moyennes des comptes effectués sur milieux non sélectifs incubés en atmosphère anaérobie (comptes anaérobies totaux cultivables: TAVC) et en atmosphère aérobie (AéroC) étaient respectivement de $42 \times 10^{4} \pm$ $94 \times 10^{4} \mathrm{CFU} / \mathrm{ml}$ (TAVC) et $17 \times 10^{4} \pm 50 \times 10^{4}$ CFU/ml (AéroC). Dans la plupart des cas (19), la flore anaérobie était prédominante (AéroC/TAVC $<1$ ). Dans le groupe 2, la flore moyenne cultivable en atmosphère anaérobie était de $1808 \times 10^{4} \pm$ $4625 \times 10^{4} \mathrm{CFU} / \mathrm{mL}$. La flore moyenne cultivable en atmosphère aérobie était de $297 \times 10^{4} \pm 692 \times$ $10^{4} \mathrm{CFU} / \mathrm{mL}$. Les prélèvements comprenaient 2 à 17 micro-organismes détectables (moyenne : $10.5 \pm 3.58$ ), sauf un prélèvement qui était monomicrobien (Actinomyces viscosus).

\section{Identification des flores}

La fréquence générale de détection des microorganismes est résumée dans le tableau 2.

Hormis les streptocoques alpha-hémolytiques (présents dans 26/26 des prélèvements du groupe 1 et dans $32 / 35$ de ceux du groupe 2), les bactéries les plus fréquemment mises en évidence étaient des anaérobies strictes (présentes respectivement dans $21 / 26$ et $32 / 35$ des prélèvements) : le genre Prevotella (15/26 et $21 / 35$ ) avec une fréquence peu différente entre Prevotellae à pigmentation noire (12/26 et $20 / 35)$ et Prevotellae non pigmentés (10/26 et 19/35), le genre Veillonella (15/26 et $11 / 35)$ et le genre capnophile Capnocytophaga (9/26 et 20/35).

Parmi les micro-organismes aéro-anaérobies, les membres des groupes Streptococcus anginosus (S. anginosus, S. constellatus, S. intermedius) (8/26 et $26 / 35)$ et Streptococcus oralis (S. gordonii, S. mitis, S. oralis, S. sanguinis) (8/26 et $22 / 35)$, ainsi que le genre Actinomyces ( $7 / 26$ et 29/35) ont été ceux qui furent les plus souvent retrouvés.

\section{Sensibilité aux antibiotiques}

Les valeurs moyennes des comptes totaux retrouvés sur milieux sélectifs pour le groupe 1 étaient en général très inférieures à celles retrouvées sur médecine

buccale

chirurgie

buccale

VOL. $10, \mathrm{~N}^{\circ} 1$ 2004

page 13 
Tableau 2 : Fréquence de détection des bactéries retrouvées (les micro-organismes sont classés selon le Bergey's Manual of Determinative Bacteriology [33]). Ces données sont extraites de celles publiées précédemment [3] [34]. Frequency of bacteria detected. (Micro-organisms are classified according do the Bergey's Manual of Determinative Bacteriology [33]). Data adapted from those previously published [3] [34].

\section{Micro-organismes}

$\begin{array}{ccc}\text { Groupe 1 } & \text { Fréquence } & \\ \mathrm{n}=26 & \text { Groupe 2 } & \text { Total } \\ & n=35 & n=61\end{array}$

\section{Anaérobies stricts}

Coques à Gram positif

Anaerococcus (Peptostreptococcus) prevotii

Micromonas (Peptostreptococcus) micros

Peptococcus niger

Peptostreptococcus anaerobius

Peptostreptococcus sp

4

Bacilles à Gram positif

Bifidobacterium spp

Clostridium spp

Eggertella (Eubacterium) lentum

médecine

buccale

chirurgie

buccale

VOL. $10, \mathrm{~N}^{\circ} 1$ 2004

page 14

Eubacterium $s p$

Mobiluncus spp

Bacilles à Gram positifs non identifiés

Coques à Gram négatif

Veillonella $s p$

3

6

Bacilles à Gram négatif

Bacteroides ovatus

Bacteroides stercoris

Bacteroides thetaiotaomicron

Bacteroides vulgatus

Bacteroides sp

Fusobacterium mortiferum

Fusobacterium necrogenes

Fusobacterium nucleatum

Leptotrichia buccalis

Prevotella buccae

Prevotella buccalis

Prevotella corporis

Prevotella bivia tannerae oeneca

Prevotella bivia disiens

Prevotella denticola

Prevotella intermedia nigrescens pallens

Prevotella loescheii

Prevotella melaninogenica

Prevotella oralis

Prevotella oris

Prevotella sp

Porphyromonas gingivalis

Bactéries à pigmentation noire non identifiées

Bacilles à Gram négatif non identifiés

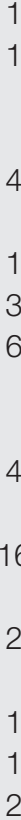


Tableau 2 (suite) :

\section{Micro-aérophiles \\ Bacilles à Gram négatif \\ Bacteroides ureolyticus \\ Campylobacter gracilis \\ Campylobacter rectus}

\section{Aérobies}

Coques à Gram positif

Enterococcus sp

Gemella haemolysans

Gemella morbillorum

Gemella sp

Lactococcus lactis cremoris

Lactococcus raffinolactis

Staphylococcus spp

Streptococcus acidominimus

Streptococcus adjacens

Streptococcus anginosus

Streptococcus constellatus

Streptococcus gordonii

Streptococcus intermedius

Streptococcus mitis

Streptococcus mutans

Streptococcus oralis

Streptococcus pyogenes

Streptococcus sanguis

Streptococcus salivarius

Streptococcus viridans

Streptococcus spp

Bacilles à Gram positif

Actinomyces gerencseriae

Actinomyces israelii

Actinomyces meyeri

Actinomyces naes/undii

Actinomyces odontolyticus

Actinomyces viscosus

Actinomyces sp

Bacillus spp

Corynebacterium afermentans

Corynebacterium bovis

Corynebacterium striatum

Corynebacterium sp

Lactobacillus acidophilus

Lactobacillus sp

Propionibacterium acnes

Propionibacterium propionicus

Propionibacterium granulosum

Bacilles à Gram négatif

Capnocytophaga spp

1

5

3

Enterobacterium $s p$

Hafnia alvei

Pasteurella multocida

Pseudomonas stutzeri 
Tableau 3 : Groupe 1 : Valeurs moyennes des comptes totaux cultivables sur milieux sélectifs et non sélectifs Group 1 : Mean values of total cultivable counts on selective and non selective media.

\begin{tabular}{|c|c|c|c|}
\hline & $\bar{x}$ & Ecart-type & Fourchette \\
\hline A1 $\left(\times 10^{4}\right)$ & 0.1023 & 0.2403 & $0-0.7393$ \\
\hline $\mathrm{A} 2\left(\times 10^{4}\right)$ & 0.073 & 0.222 & $0-0.148$ \\
\hline$P\left(x 10^{4}\right)$ & 0.1557 & 0.3389 & $0-1.6$ \\
\hline$S\left(x 10^{4}\right)$ & 0.4207 & 0.4789 & $0.0179-1.64$ \\
\hline$M\left(x 10^{4}\right)$ & 0.8084 & 0.8358 & $0.0103-2.9467$ \\
\hline $\operatorname{SM}\left(x 10^{4}\right)$ & 0.4339 & 0.4624 & $0.0182-1.64$ \\
\hline \multicolumn{2}{|c|}{$\begin{array}{l}\text { Flore Totale Anaérobie Cultivable = FTAC } \\
\text { AeroC Flore Totale Aérobie Cultivable } \\
\text { A1 Gélose au sang + Amoxicilline }(0.5 \mathrm{mg} / \mathrm{ml}) \\
\text { A2 Gélose au sang + Amoxicilline }(4 \mathrm{mg} / \mathrm{ml}) \\
\text { P Gélose au sang + Pristinamycine }(1 \mathrm{mg} / \mathrm{ml}) \\
\text { S Gélose au sang + Spiramycine }(1 \mathrm{mg} / \mathrm{ml}) \\
\text { M Gélose au sang + Métronidazole }(4 \mathrm{mg} / \mathrm{ml}) \\
\text { SM Gélose au sang + Spiramycine } / \text { Métronidazole }(1 / 4 \mathrm{mg} / \mathrm{ml})\end{array}$} & \multicolumn{2}{|c|}{$\begin{array}{l}\text { Total Anaerobic Viable Count }=\text { TAVC } \\
\text { AeroC Total Aerobic Viable Count } \\
\text { A1 Blood agar + Amoxicillin }(0.5 \mathrm{mg} / \mathrm{ml}) \\
\text { A2 Blood agar + Amoxicillin }(4 \mathrm{mg} / \mathrm{ml}) \\
\text { P Blood agar + Pristinamycin }(1 \mathrm{mg} / \mathrm{ml}) \\
\text { S Blood agar + Spiramycin }(1 \mathrm{mg} / \mathrm{ml}) \\
\text { M Blood agar + Metronidazole }(4 \mathrm{mg} / \mathrm{ml}) \\
\text { SM Blood agar + Spiramycin } / \text { Metronidazole }(1 / 4 \mathrm{mg} / \mathrm{ml})\end{array}$} \\
\hline
\end{tabular}

médecine buccale chirurgie buccale

VOL. $10, \mathrm{~N}^{\circ} 1$ 2004

page 16

Tableau 4 : Groupe 1: Rapports moyens [Compte total sur milieu sélectif] divisé par FTAC Group 1 : Mean ratio [Total Count on selective medium] divided by TAVC

\begin{tabular}{|c|c|c|c|}
\hline & Moyenne & Ecart-type & Fourchette \\
\hline A1 / TAVC & 0.059 & 0.149 & $0-0.739$ \\
\hline A2 / TAVC & 0.040 & 0.122 & $0-0.542$ \\
\hline P / TAVC & 0.078 & 0.104 & $0-0.278$ \\
\hline S / TAVC & 0.256 & 0.289 & $0-1.08$ \\
\hline M / TAVC & 0.566 & 0.704 & $0-2.174$ \\
\hline SM / TAVC & 0.347 & 0.387 & $0-1.753$ \\
\hline
\end{tabular}

Tableau 5 : Groupe 1 : Fréquence de détection des micro-organismes selon les milieux utilisés. Group 1 : Frequency of micro-organisms detected on various selective media

$\begin{array}{lccccccc}\text { Micro-organismes } & \text { A1 } & \text { A2 } & \text { P } & \text { S } & \text { M } & \text { SM } & \text { Sujets porteurs } \\ \text { Actinomyces israelii } & 1 & 0 & 0 & 1 & 1 & 1 & 5 \\ \text { Bacteroides spp } & 3 & 3 & 3 & 0 & 0 & 0 & 9 \\ \text { Capnocytophaga spp } & 3 & 3 & 0 & 2 & 3 & 2 & 9 \\ \text { Gemella spp } & 1 & 1 & 1 & 3 & 1 & 2 & 8 \\ \text { Prevotellae non pigmentés } & 8 & 6 & 3 & 2 & 0 & 0 & 9 \\ \text { Prevotella intermedia * } & 6 & 7 & 0 & 2 & 0 & 0 & 9 \\ \text { Porphyromonas gingivalis } & 0 & 2 & 0 & 2 & 0 & 0 & 3 \\ \text { Staphylococcus sp } & 2 & 1 & 3 & 1 & 4 & 1 & 8 \\ \text { Streptococcus anginosus (groupe) } & 2 & 1 & 0 & 4 & 2 & 3 & 8 \\ \text { Veillonella sp } & 4 & 1 & 11 & 6 & 1 & 0 & 16 \\ \text { Levures } & 2 & 2 & 0 & 0 & 2 & 0 & 5\end{array}$

* groupe Prevotella intermedia: P. intermedia/nigrescens/pallens 
milieux non sélectifs (Tableau 3). Les moyennes des rapports compte sur milieu sélectif sur compte anaérobie total sont résumées dans le tableau 4. Dans respectivement un (A1), cinq (A2) et un $(P)$ - c'est-à-dire des milieux contenant un antibiotique -, aucun micro-organisme n'a été détecté.

La fréquence de détection sur les différents milieux sélectifs du groupe 1 est résumée dans le tableau 5. Aucune bactérie anaérobie stricte n'a été détectée dans respectivement 4 (A1), 6 (A2), 7 (P), $12(\mathrm{~S}) ; 20(\mathrm{M})$ et 20 (SM) des 26 cas. Quarante-quatre souches issues de milieux contenant l'amoxicilline ont été testées pour la production de bêta-lactamases. Dix-huit souches, provenant de neuf individus, présentaient une réponse positive : bactéries des genres Prevotella (9), Bacteroides (3), Staphylococcus (4), Capnocytophaga (1) et Fusobacterium (1). La résistance à l'amoxicilline de ces dix-huit souches a été confirmée par dilution en gélose.

Tous les anaérobies stricts testés parmi les bactéries isolées dans le groupe 2 (63 souches) ont montré une sensibilité à $\mathrm{M}, \mathrm{MS} 1$ et $\mathrm{MS} 2$, tandis que la sensibilité à $A 1$ et $A 2$ était retrouvée chez respectivement 47 et 52 souches. La pristinamycine s'est révélée efficace sur tous les anaérobies stricts sauf les Veillonella, et sur tous les anaérobies facultatifs testés (157 souches). La sensibilité à $A 1$ a été retrouvée chez 146 souches anaérobies facultatives, tandis que 119 souches se révélaient sensibles à MS2. La production de bêta-lactamases a été détectée au niveau de six souches (2 de staphylococci et 4 de Prevotellae) issues de 5 prélèvements.

\section{DISCUSSION}

Les péricoronarites des troisièmes molaires mandibulaires sont des affections inflammatoires et infectieuses de l'adulte jeune dont les manifestations locales et générales sont extrêmement variables d'un sujet à l'autre. Les pseudo-poches distales peuvent créer des conditions locales d'anaérobiose favorisant le développement d'une flore particulière. Les résultats obtenus dans les deux groupes confirment le caractère anaérobie de la flore. Les bactéries anaérobies strictes sont détectées dans $87 \%$ des échantillons (53/61). Les bactéries micro-aérophiles (B. ureolyticus, genre Campylobacter) et/ou à croissance préférentiellement anaérobie (genres Capnocytophaga, Actinomyces...) sont retrouvées dans trois des huit autres échantillons. Parmi les bacétries anaérobies, notons le taux de détection non négligeable des bactéries du genre Clostridium dans plus d'un cas sur dix (7/61), dont un C. perfringens, bactérie retrouvée dans des formes de gangrène particulièrement destructrices.

Ces résultats corroborent ceux parus dans la littérature à l'aide de sondes ADN [6], de milieux non sélectifs [7, 8] ou sélectifs [9-11].

Malgré ces conditions proches de celles des poches parodontales, notamment la dérive anaérobie, les pathogènes parodontaux majeurs ne sont pas ou peu rencontrés: Actinobacillus actinomycetemcomitans (0/61), Tannerella (Bacteroides) forsythus (0/61), Porphyromonas gingivalis (4/61). Le taux de détection de ces bactéries varie selon les études [6-10,12]. Des variations dans les populations et les méthodes utilisées peuvent expliquer ces différences. Les pathogènes parodontaux les plus fréquemment retrouvés dans cette étude ont été le groupe $P$. intermedia/nigrescens/pallens (23/61), Eggertella (Eubacterium) lentum (11/61), Campylobacter rectus (11/61), Micromonas (Peptostreptococcus) micros (10/61), Fusobacterium nucleatum (8/61). Ces micro-organismes font partie d'un groupe homogène de pathogènes parodontaux, appelé groupe orange, significativement associé à une augmentation de profondeur des poches [13, 14]. Ceux-ci sont souvent étroitement associés les uns aux autres dans les lésions de parodontites. Lors de ces pathologies, l'administration par voie générale de métronidazole permet de diminuer leur quantité et d'améliorer l'état parodontal [13, 14]. Actinomyces israelii a été détecté dans près de un cas sur trois (20/61). De même que d'autres actinomycètes ou le genre Propionibacterium, $A$. israelii peut être associé à des gingivites. Ce germe est également la principale bactérie retrouvée dans l'actinomycose cervico-faciale, dans une flore polymicrobienne associant des bacilles à Gram positif (Actinomyces, Propionobacterium), des bacilles anaérobies à Gram négatif médecine

buccale

chirurgie

buccale

VOL. $10, \mathrm{~N}^{\circ} 1$ 2004

page 17 
médecine

buccale

chirurgie

buccale

VOL. $10, \mathrm{~N}^{\circ} 1$ 2004

page 18
(Prevotellae pigmentés en noir, P. gingivalis...), voire des streptocoques [15]. La présence de ces bactéries dans plusieurs échantillons suggère que les phénomènes pathogéniques pourraient, dans certains cas de péricoronarites, être proches de ceux des actinomycoses.

Les streptocoques buccaux (alpha-hémolytiques ou viridans) ont été les micro-organismes les plus fréquemment détectés $(58 / 61)$. Ce sont pour la plupart des bactéries commensales de la cavité buccale mais elles peuvent être responsables d'infections systémiques ou d'abcès localisés [16-18]. Le taux de détection élevé s'explique probablement en partie par le fait que les pseudo-poches des péricoronarites sont ouvertes sur la cavité buccale, autorisant une colonisation régulière depuis la salive. Les dents de sagesse en éruption constituent un réservoir pour ces bactéries et les péricoronarites pourraient favoriser des infections ORL comme les angines [12] et des complications infectieuses à distance comme l'endocardite. Les streptocoques viridans comprennent plusieurs groupes dont ceux des groupes oralis, mitis et du groupe anginosus. Les streptocoques du groupe anginosus sont régulièrement associés à des abcès ou d'autres infections, notamment à flore polymicrobienne mixte aéro-anaérobie. Ils ont récemment été suspectés d'être des pathogènes majeurs pour les péricoronarites [9].

Les antibiotiques utilisés dans cette étude correspondent à ceux les plus régulièrement prescrits par les praticiens français [19, 20] auxquels a été ajoutée la pristinamycine, antibiotique recommandé pour les patients à haut risque infectieux, mais qui sont allergiques à l'amoxicilline [21]. Leurs spectres d'action recouvrent la majorité des genres et espèces de la cavité buccale.

L'amoxicilline et la pristinamycine ont été les antibiotiques les plus actifs sur la flore totale cultivable en anaérobiose (Tableau 3).

L'amoxicilline est un antibiotique à large spectre, actif aussi bien sur la flore anaérobie stricte que sur la flore anaérobie facultative. Son utilisation entraîne la sélection de germes résistants naturels (Veillonella sp) ou acquis (souches productrices de bêta-lactamases). Les souches productrices de bêta-lactamases peuvent être présentes dans la cavité buccale dès le plus jeune âge [22], et elles peuvent être associées à divers types de pathologies infectieuses buccales [23-29]. Leur fréquence varie selon les populations [25]. Des souches productrices de bêta-lactamases ont été retrouvées dans $34,6 \%$ des prélèvements du premier groupe et $14 \%$ du deuxième groupe. Elles appartiennent essentiellement à trois genres bactériens : deux genres anaérobies stricts (Prevotella et Bacteroides) et un genre aéro-anaérobie (Staphylococcus). Ces genres sont les producteurs de bêta-lactamases les plus fréquents dans la cavité buccale [24, 30,31]. Ces résultats suggèrent l'existence d'une pression de sélection liée à l'emploi de la pénicilline dès le plus jeune âge, et ils soulignent le risque d'échec d'une antibiothérapie à base de pénicilline lorsque les souches productrices de bêta-lactamases sont présentes. La pristinamycine était active sur toutes les souches productrices de bêta-lactamases du groupe 1 et sur 206 des 211 souches testées dans le groupe 2 (aérobies et anaérobies confondues). Seules les Veillonella étaient résistantes, ce qui confirme les données d'une précédente étude [32]. Ces résultats montrent que la pristinamycine est un antibiotique à forte activité et présentant peu de résistances. Son spectre le rend particulièrement intéressant dans les infections aiguës et lors d'échecs avec d'autres antibiotiques. II confirme l'intérêt de la pristinamycine chez les patients à haut risque infectieux.

Le métronidazole, seul ou associé à la spiramycine, a montré la plus grande efficacité sur la flore anaérobie stricte, tout en respectant mieux les bactéries aéro-anaérobies faisant partie de la flore habituelle de la cavité buccale. La prescription du métronidazole présente donc un intérêt lors de péricoronarites liées à une flore anaérobie sécrétrice de bêta-lactamases.

Cette étude a mis en évidence l'hétérogénéité des péricoronarites et la nature complexe et mixte, aéro-anaérobie, de la flore microbienne qui y est associée ; la flore anaérobie étant prédominante. L'amoxicilline et la pristinamycine ont été les antibiotiques les plus actifs sur la flore totale cultivable, notamment sur les bactéries aérobies. Cependant, des souches porteuses de 
bêta-lactamases ont été rencontrées chez plus du tiers des sujets étudiés : elles représentent un risque d'échec en cas d'antibiothérapie utilisant seulement des bêta-lactamines seules. Le métronidazole, seul ou l'association spiramycine- métronidazole, a été le plus efficace sur les anaérobies et le moins agressif sur la flore non anaérobie stricte qui constitue la majeure partie de la flore normale de la cavité buccale.

\section{RÉFÉRENCES}

1 - De Mello G, Sixou Jl, Jeandot J, Duran D, Feki A, ROCHE Y, DUBREUIL L. Etude comparative de l'association spiramycine-métronidazole et de l'amoxicilline dans le traitement des péricoronarites de l'adulte. Med Buccale Chir Buccale 2003; 9: 159-65.

2 - Dahlèn G, Pipattanagovit P, Rosling B, Moller AJ. A comparison of two transport media for saliva and subgingival samples. Oral Microbiol Immunol 1993; 8: 37582.

3 - Sixou JL, Magaud C, Jolivet-Gougeon A, Cormier M, BonnAURE-Mallet M. Microbiology of mandibular third molar pericoronitis: Incidence of beta-lactamase-producing bacteria. Oral Surg Oral Med Oral Pathol Oral Radiol Endod 2003; 95: 655-9.

4 - National Committee for Clinical and Laboratory Standards. Methods for antimicrobial susceptibility testing of anaerobic bacteria: approved standard. Standard Wayne NCCLS; $5^{\text {th }}$ ed, 2001 (36 pp).

5 - Communiqué 2002 du Comité de l'Antibiogramme de la Société Française de Microbiologie. Société Française de Microbiologie, Paris, 2002 (31 pp).

6 - Blakey GH, White RP JR, Offentacher S, Phillips C, Delano EO, MAYNOR G. Clinical/biological outcomes of treatment for pericoronitis. J Oral Maxillofac Surg 1996; 54: 1150-60.

7 - Leung WK, Theilade E, Comfort MB, Lim PL. Microbiology of the pericoronal pouch in mandibular third molar pericoronitis. Oral Microbiol Immunol 1993; 8: 306-12.

8 - Wade WG, Gray AR, Absi EG, BARKER GR. Predominant cultivable flora in pericoronitis. Oral Microbiol Immunol 1991; 6: 310-2.

9 - Peltroche-Llacsahuanga H, Reichhart E, Schmitt W, LUTTICKEN R, HAASE G. Investigation of infectious organisms causing pericoronitis of the mandibular third molar. J Oral Maxillofac Surg 2000; 58: 611-6.

10 - Mombelli A, Buser D, Lang NP, Berthold H. Suspected periodontopathogens in erupting third molar sites of periodontally healthy individuals. J Clin Periodontol 1990; 17: 48-54.

11 - Mombelli A. Microbiology and antimicrobial therapy of peri-implantitis. Periodontol 2000 2002; 28: 177-89.

12 - Rajasuo A, Jousimies-Somer H, SAVOlainen S, Leppanen J, MurtomaA H, Meurman JH. Bacteriologic findings in tonsillitis and pericoronitis. Clin Infect Dis 1996; 23: 51-60.
13 - Socransky SS, Haffajee AD, Cugini MA, Smith C, KENT RL JR. Microbial complexes in subgingival plaque. J Clin Periodontol 1998; 25: 134-44.

14 - Socransky SS, HafFAJEE AD. Dental biofilms: difficult therapeutic targets. Periodontol 2000 2002; 28: 12-55.

15 - Jousimies-SOmer HR, Summanen PH, Finegold SM. Bacteroides, Porphyromonas, Prevotella, Fusobacterium, and other anaerobic Gram-negative rods and cocci (p. 690-711). In: P.R. Murray, E.J. Baron, M.A. Pfaller, F.C. Tenover, R.H. Yolken, eds. Manual of clinical microbiology. ASM Press, Washington DC 1999.

16 - Tomas Carmona I, Diz Dios P, Scully C. An update on the controversies in bacterial endocarditis of oral origin. Oral Surg Oral Med Oral Pathol Oral Radiol Endod 2002; 93: 660-70.

17 - Su TM, LIN YC, LU CH, Chang WN, LILIANG PP, RAu CS, LIANG CL, TSAI YD, LEE TJ, CHEN HJ. Streptococcal brain abscess: analysis of clinical features in 20 patients. Surg Neurol 2001; 56: 189-94.

18 - STосK JH, SAHN DJ. Endocarditis in the pediatric population. Curr Treat Options Cardiovasc Med 2000; 2: 481-8.

19 - Matysiak M, Gradelet J, Mignée MJ, Mabriez JC. Evaluation des prescriptions pharmacologiques des chirurgiens-dentistes de la circonscription de la CPAM de Grenoble. Rev Méd Assurance Maladie 1997: 71-88.

20 - Antibiotiques. Les prescriptions en question. Lettre Chir Dent 2002; 6: 15.

21 - Prescription des antibiotiques en odontologie et stomatologie. Recommandations et argumentaire. Agence Française de Sécurité Sanitaire des Produits de Santé. Méd Mal Infect 2002; 32: 125-60.

22 - KönÖnen E, KanerVo A, TAKAla A, Asikainen S, JousimiESSOMER $H$. Establishment of oral anaerobes during the first year of life. J Dent Res 1999; 78: 1634-9.

23 - van Winkelhoff AJ, Winkel EG, BarendREgt D, Dellemijn-Kippuw N, Stijne A, van der Velden U. BetaLactamase producing bacteria in adult periodontitis. J Clin Periodontol 1997; 24: 538-43.

24 - Fosse T, Madinier I, HitZIG C, Charbit Y. Prevalence of beta-lactamase-producing strains among 149 anaerobic gram-negative rods isolated from periodontal pockets. Oral Microbiol Immunol 1999; 14: 352-7.

25 - HerRera D, van WinKelhoff AJ, DellemiJn-Kippuw N, WINKEL EG, SANZ M. Beta-lactamase producing bacte-

\begin{tabular}{l}
$\begin{array}{l}\text { médecine } \\
\text { buccale } \\
\text { chirurgie } \\
\text { buccale }\end{array}$ \\
\hline vOL. $10, N^{\circ} 1$ \\
2004 \\
\hline page 19
\end{tabular}

page 19 
médecine buccale chirurgie buccale

VOL. $10, \mathrm{~N}^{\circ} 1$ 2004 ria in the subgingival microflora of adult patients with periodontitis. A comparison between Spain and the Netherlands. J Clin Periodontol 2000; 27: 520-5.

26 - Kuriyama T, Karasawa T, Nakagawa K, SaIKI $Y$, Yamamoto E, NaKAMURA S. Bacteriologic features and antimicrobial susceptibility in isolates from orofacial odontogenic infections. Oral Surg Oral Med Oral Pathol Oral Radiol Endod 2000; 90: 600-8.

27 - KurIYAMa T, NaKagaWA K, Karasawa T, SAIKI Y, YAMAMOTO E, NAKAMURA S. Past administration of betalactam antibiotics and increase in the emergence of beta-lactamase-producing bacteria in patients with orofacial odontogenic infections. Oral Surg Oral Med Oral Pathol Oral Radiol Endod 2000; 89: 186-92.

28 - Kuriyama T, Karasawa T, Nakagawa K, Yamamoto E, NAKAMURA S. Incidence of beta-lactamase production and antimicrobial susceptibility of anaerobic gram-negative rods isolated from pus specimens of orofacial odontogenic infections. Oral Microbiol Immunol 2001; 16: 10-5.

29 - Kuriyama T, Karasawa T, Nakagawa K, Yamamoto E, NAKAMURA S. Bacteriology and antimicrobial susceptibility of gram-positive cocci isolated from pus specimens of orofacial odontogenic infections. Oral Microbiol Immunol 2002; 17: 132-5.
30 - Nyfors S, Kononen E, TAKala A, Jousimies-SOMER H. Beta-lactamase production by oral anaerobic gramnegative species in infants in relation to previous antimicrobial therapy. Antimicrob Agents Chemother 1999; 43: 1591-4.

31 - Fosse T, Madinier I, Hannoun L, Giraud-Morin C, HITZIG C, CHARBIT Y, OURANG S. High prevalence of cfXA beta-lactamase in aminopenicillin-resistant Prevotella strains isolated from periodontal pockets. Oral Microbiol Immunol 2002; 17: 85-8.

32 - DUBREUIL L, HOUCKE I, SINGER E. In vitro activity of 10 antibiotics including pristinamycin and its two components (RP 12536 and 27404) against strict anaerobes. Pathol Biol 1998; 46: 147-52.

33 - Holt JG, KRieg NR, Sneath PHA, Staley JT, WillaAms ST. Bergey's manual of determinative bacteriology (pp 1 787), $9^{\text {th }}$ ed. Williams \& Wilkins, Philadelphia, 1994.

34 - Sixou JL, Magaud C, Jolivet-Gougeon A, Cormier M, BONNAURE-MALLET M. Evaluation of mandibular third molar pericoronitis flora and its susceptibility to different antibiotics prescribed in France. J Clin Microbiol 2003; 41: 5794-97. 